\title{
Arthroscopic verification of objectivity of the orthopaedic examination and magnetic resonance imaging in intra-articular knee injury. Retrospective study
}

\author{
Julian Dutka, Michał Skowronek, Paweł Skowronek, Łukasz Dutka \\ Department of Orthopedic and Traumatologic Surgery, Zeromski Hospital, Cracow, Poland
}

Videosurgery and Other Miniinvasive Techniques 2012; 7 (1): 13-18 DOI: $10.5114 /$ wiitm.2011.25638

\begin{abstract}
Introduction: Arthroscopy of the knee joint is regarded as the most objective diagnostic method in intra-articular knee joint lesions.

Aim: The purpose of this study was to assess the objectivity and diagnostic value of orthopaedic examination (OE) and magnetic resonance imaging (MRI) in reference to the arthroscopic result.

Material and methods: In a group of 113 patients treated by arthroscopic surgery for post-traumatic knee pathology between 2008 and 2010 in our department, accuracy of clinical and MRI findings that preceded surgery were studied retrospectively using a statistical method. Sensitivity, specificity, accuracy and predictive negative and positive values were the subject of analysis.

Results: In the presented trial, sensitivity values of the orthopaedic examination for injuries of the anterior cruciate ligament $(A C L)$, meniscus medialis (MM), meniscus lateralis $(M L)$ and chondral injuries (Chl) were $86 \%, 65 \%$, 38\% and $51 \%$, respectively. Specificity values were $90 \%, 65 \%, 100 \%$ and $100 \%$, respectively. The MR sensitivity and specificity values were $80 \%, 88 \%, 44 \%$ and $32 \%$, and $86 \%, 64 \%, 93 \%$ and $97 \%$, respectively.

Conclusions: Assessment of intra-articular knee joint lesions is a difficult diagnostic problem. In making a decision about arthroscopy of the knee joint, an appropriate sequence of examinations should be carried out: OE, MRI and arthroscopy. The improvement in the effectiveness of the orthopaedic examination and MRI can limit the too high frequency of diagnostic arthroscopies, which generates the risk of operation treatment and costs.
\end{abstract}

Key words: arthroscopy, orthopaedic examination, magnetic resonance imaging, knee injury.

\section{Introduction}

Magnetic resonance imaging (MRI) of the knee joint is regarded as the gold standard in the diagnostics of knee injuries, and since the 1980s it has become more and more popular [1]. The MRI is regarded as an alternative examination with respect to diagnostic arthroscopy. In clinical practice MRI is also regarded as a more credible examination than orthopaedic examination (OE) and it very often pre- cedes arthroscopy of the knee joint. A treatment dilemma occurs when clinical examination and MRI contradict each other. But is it justified when the orthopaedic examination confirms a knee lesion requiring arthroscopy? And if the MRI diagnosis turns out to be false, will we withdraw the patient from the operation? The MRI is expensive and still not widely available, which can also delay definitive treatment. Still there is no unanimous opinion concerning the credibility of diagnosis based on 
MRI compared with the ultimate and credible arthroscopy verification.

\section{Aim}

The purpose of this study was to assess the objectivity and diagnostic value of OE and MRI in reference to the arthroscopic result.

\section{Material and methods}

A retrospective trial was conducted including patients with suspected knee lesions and qualified for arthroscopy based on OE or MRI. Between 2008 and 2010 arthroscopy was carried out in 208 patients, with 158 of them having MRI examination done. From this group, 113 met inclusion criteria as follows: had knee injury, were diagnosed with OE and $M R I$ and were treated with arthroscopy. Patients with articular surface fractures, presence of loose bodies and arthrosis of the knee joint were excluded from the study. Patients who had knee injury after MRI assessment were also excluded. The X-ray examination was to detect bone fractures and it was not assessed in further analysis. All patients before arthroscopy of the knee joint were examined by experienced orthopaedists. The orthopaedic examination entailed the assessment of damaged intraarticulate structures of the knee: crucial ligaments (anterior cruciate ligament - ACL, posterior cruciate ligament $-\mathrm{PCL}$ ), meniscuses (medialis $-M M$, lateralis - ML) and chondral defects of the joint surface. Orthopaedic surgeons used the same protocol of the examination including clinical tests of the assessment of individual structures of the knee: anterior and posterior drawer test, Lachman, pivot-shift,

Table I. Demographic data

\begin{tabular}{|lc|}
\hline No. of patients & 113 \\
\hline Age (SD) [years] & $37.2(12.2)$ \\
\hline ACL lesion [\%] & 71 \\
\hline MM lesion [\%] & 43 \\
\hline ML lesion [\%] & 16 \\
\hline Chondral defect [\%] & 41 \\
\hline PCL lesion [\%] & 1 \\
\hline
\end{tabular}

$A C L$ - anterior cruciate ligament, $M M$ - meniscus medialis, $M L$ - meniscus lateralis, $P C L$ - posterior cruciate ligament
McMurey's and Apley tests [2]. The MRI of the knee joint was done in various centres not later than 1 month before arthroscopy and reported by radiologists who knew the preliminary diagnosis. All arthroscopies were done as part of the hospitalization by 2 experienced surgeons. Arthroscopy was performed from at least two ports for accurate visualisation of articular structures and the possibility of surgical intervention. Joint cartilage was assessed according to the Outerbridge criteria [3]. Injuries of crucial ligaments were identified when total or partial interruption was found and confirmed with dynamic examination. Meniscal lesions were diagnosed in stress positions using a probe device. All pathological lesions were registered (print, video). The statistical analysis compared orthopaedic examination and MRI findings with the result of arthroscopy. To determine the credibility of the examinations and MRI sensitivity, the specificity, positive predictive value (PPV), negative predictive value (NPV) and accuracy were assessed. Significance was determined at $p<0.05$.

\section{Results}

In 113 patients knee injuries were diagnosed on orthopaedic examination and MRI followed by arthroscopy. Mean age of the patients was 37.2 years. Demographic data are presented in Table I. In all arthroscopies, $71 \mathrm{ACL}$ lesions, $1 \mathrm{PCL}, 43 \mathrm{MM}, 16 \mathrm{ML}$ and 41 chondral defects were diagnosed (Table I). The sensitivity of MRI and orthopaedic examination for ACL were $80 \%$ and $86 \%$ and no statistical differences were observed. The specificity was $86 \%$ and $90 \%$ and statistically significant differences were not observed (Table II). For MM lesions MRI sensitivity was $88 \%$

Table II. Results of the data analysis: sensitivity, specificity, PPV, NPV, accuracy for ACL lesions

\begin{tabular}{|lccc|}
\hline Parameter & MRI & OE & Value of $p$ \\
\hline Sensitivity $(95 \% \mathrm{Cl})$ & $80(69-89)$ & $86(76-93)$ & 0.370 \\
\hline Specificity $(95 \% \mathrm{Cl})$ & $86(71-95)$ & $90(77-97)$ & 0.500 \\
\hline PPV $(95 \% \mathrm{Cl})$ & $90(80-96)$ & $94(85-99)$ & 0.478 \\
\hline NPV $(95 \% \mathrm{Cl})$ & $72(58-84)$ & $79(65-90)$ & 0.410 \\
\hline Accuracy $(95 \% \mathrm{Cl})$ & $82(74-89)$ & $88(80-93)$ & 0.264 \\
\hline
\end{tabular}

$P P V$ - positive predictive value, NPV - negative predictive value, MRI-magnetic resonance imaging, $O E$-orthopaedic examination 
Table III. Results of the data analysis: sensitivity, specificity, PPV, NPV, accuracy in MM lesions

\begin{tabular}{|lccc|}
\hline Parameter & MRI & OE & Value of $p$ \\
\hline Sensitivity $(95 \% \mathrm{Cl})$ & $88(75-96)$ & $65(49-79)$ & 0.011 \\
\hline Specificity $(95 \% \mathrm{CI})$ & $64(52-75)$ & $87(77-94)$ & 0.002 \\
\hline PPV $(95 \% \mathrm{Cl})$ & $60(47-72)$ & $76(59-88)$ & 0.118 \\
\hline NPV $(95 \% \mathrm{Cl})$ & $90(78-97)$ & $80(70-89)$ & 0.143 \\
\hline Accuracy $(95 \% \mathrm{Cl})$ & $73(64-81)$ & $79(70-86)$ & 0.349 \\
\hline
\end{tabular}

Abbreviations - see table II

and for orthopaedic examination $65 \%$; this difference is statistically significant to the benefit of MRI. However, the specificity for orthopaedic examination was $87 \%$ and for MRI $64 \%$; the difference is statistically significant (Table III). Sensitivity of the resonance in the case of ML lesions was $44 \%$ and the OE $38 \%$; differences between examinations are not statistically significant. For ML lesions the OE is more specific and was $100 \%$, for MRI $93 \%$, and this difference is statistically significant (Table IV). Differences of specificity for cartilage lesions in MRI and OE did not demonstrate statistical differences and they were $32 \%$ and $51 \%$ respectively. The specificity for these lesions did not show statistical differences but they demonstrate much greater values and the score for MRI was $97 \%$ and for OE 100\% (Table V). Because of the very few cases of PCL lesions, statistical analyses were not carried out. In the studied material 1 PCL was diagnosed in arthroscopy and confirmed in MRI but not in the OE. In 1 MRI, a PCL lesion was diagnosed which was not confirmed in arthroscopy and OE. The credibility of sensitivity and the specificity of diagnostic tests for intra-articular structures were additionally confirmed with PPV, NPV and accuracy results.

\section{Discussion}

Assessment of intra-articular lesions is a difficult problem in diagnostics of injuries of the knee joint. In knee injury very often more than one structure is damaged and it may complicate establishing the final and precise diagnosis. The purpose of this study is to assess the value and the diagnostic usefulness of $O E$ and $M R I$ in the diagnostics of intra-articular knee lesions. The MRI is a very often carried out and abused examination in many cases. The MRI is wrongfully regarded as the most precise and referen-
Table IV. Results of the data analysis: sensitivity, specificity, PPV, NPV, accuracy in ML lesions

\begin{tabular}{|lccc|}
\hline Parameter & MRI & OE & Value of $p$ \\
\hline Sensitivity $(95 \% \mathrm{Cl})$ & $44(20-70)$ & $38(15-65)$ & 0.719 \\
\hline Specificity $(95 \% \mathrm{Cl})$ & $93(86-97)$ & $100(96-100)$ & 0.007 \\
\hline PPV $(95 \% \mathrm{Cl})$ & $50(23-77)$ & $100(54-100)$ & 0.032 \\
\hline NPV $(95 \% \mathrm{Cl})$ & $91(83-96)$ & $91(83-95)$ & 0.950 \\
\hline Accuracy $(95 \% \mathrm{Cl})$ & $86(78-92)$ & $91(84-96)$ & 0.211 \\
\hline Abbreviations - see table II & & & \\
\hline
\end{tabular}

tial in establishing the accurate diagnosis. However, MRI did not lower the rank of the orthopaedic examination indications for arthroscopy, which gives the credible diagnosis and the possibility of precise treatment.

The ACL lesions are often reliably confirmed both in MRI and OE (Figures 1-2). The sensitivity and specificity of diagnostic methods in the present study are similar. Therefore MRI in ACL lesions is not a referential examination and is not necessary in making a decision in arthroscopy. In the case of lack of manifestations from other intra-articular damaged structures, the $\mathrm{OE}$ and medical interview give enough information to establish further treatment. Rayan et al. [4] presented similar results of the MRI and OE sensitivity, respectively $81 \%$ and $77 \%$. Many other authors have the same point of view [5]. They also claim that the accurate $\mathrm{OE}$ is an effective tool in qualification of the patient for $A C L$ reconstruction.

The MM lesions are a frequent injury and sensitivity of MRI in our material is greater than OE. However, OE shows greater specificity. In the assessment of MM lesions, MRI seems to be more justified taking

Table V. Results of the data analysis: sensitivity, specificity, PPV, NPV, accuracy in chondral defects

\begin{tabular}{|lccc|}
\hline Parameter & MRI & OE & Value of $p$ \\
\hline Sensitivity $(95 \% \mathrm{CI})$ & $32(18-48)$ & $51(35-67)$ & 0.073 \\
\hline Specificity $(95 \% \mathrm{Cl})$ & $97(90-100)$ & $100(95-100)$ & 0.154 \\
\hline PPV (95\% Cl) & $87(60-98)$ & $100(84-100)$ & 0.085 \\
\hline NPV $(95 \% \mathrm{Cl})$ & $71(61-80)$ & $78(84-100)$ & 0.279 \\
\hline Accuracy $(95 \% \mathrm{Cl})$ & $73(64-81)$ & $82(74-89)$ & 0.109 \\
\hline
\end{tabular}

Abbreviations - see table II 


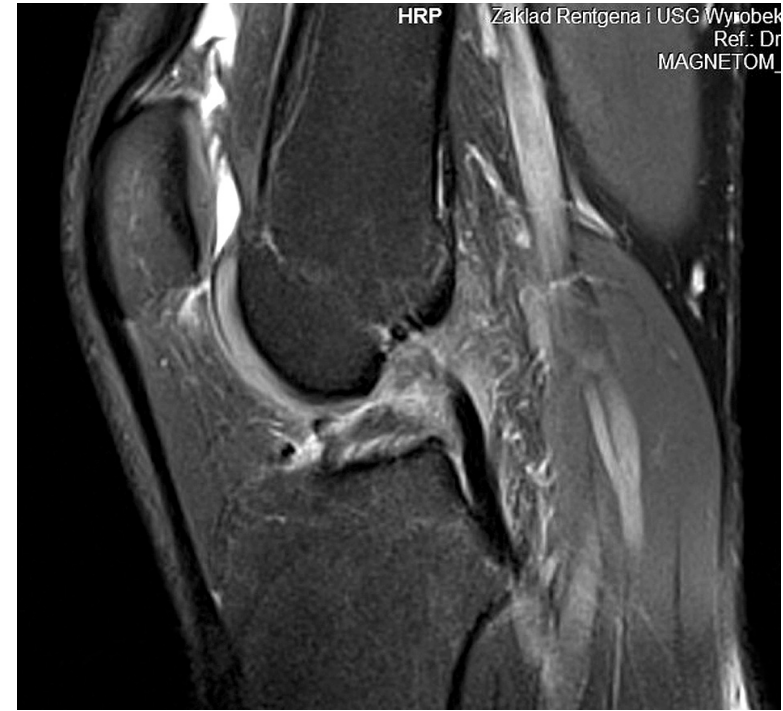

Figure 1. The ACL lesion in MRI

into consideration the diversity of morphology of the lesions (Figures 3-4). Kuikka et al. [6] in acute MM tears reported sensitivity of MRI of $91.7 \%$ and specificity of $87.1 \%$; however, they did not compare results with OE. In a multi-centre study, Fisher et al. [7] assessed the medium sensitivity of MRI as $93 \%$ and specificity as $84 \%$. But research findings between centres differed (sensitivity $80-94 \%$, specificity 58 -

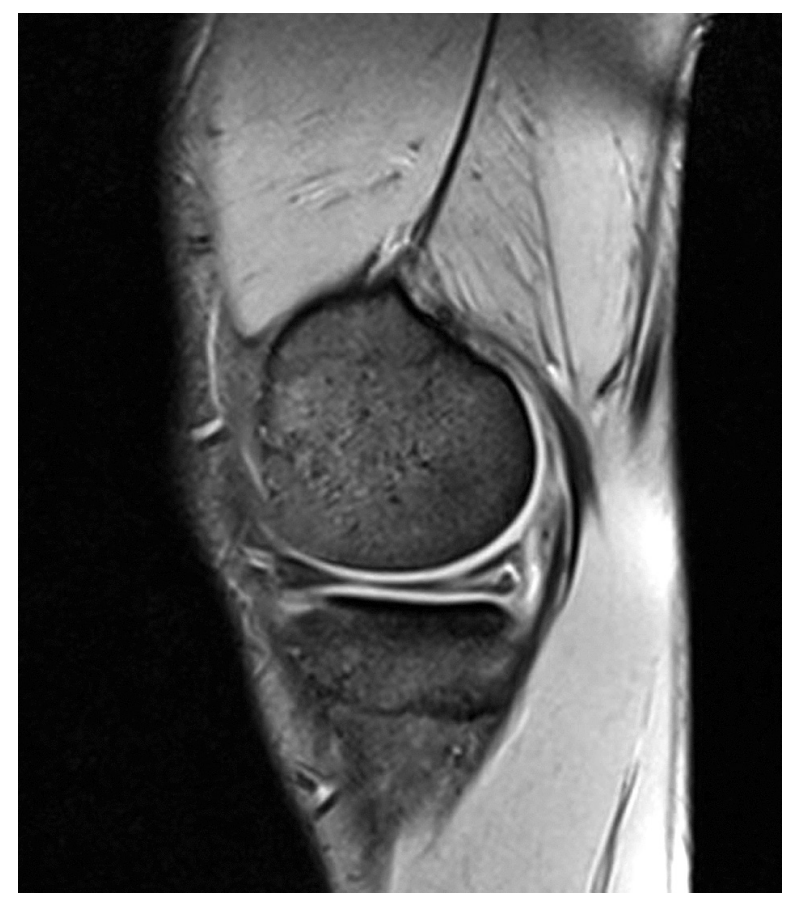

Figure 3. The MM posterior horn lesion in MRI

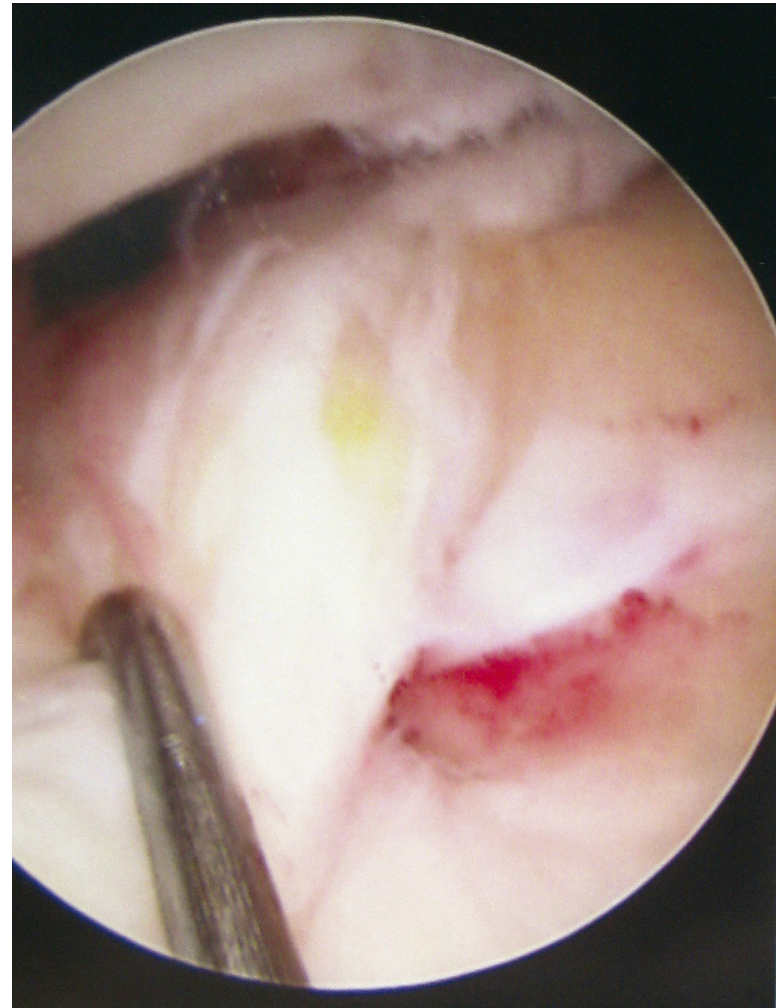

Figure 2. Arthroscopy verification of ACL lesion

$85 \%)$, as is also presented in other authors' trials [8, 9]. Rose et al. [10] compared MRI with the OE and they stated that they had equivalent value. The variations of sensitivity and specificity of MRI in publications gives evidence of MRI over-interpretation, which arthroscopy verified. In this aspect the value of OE should again be emphasized.

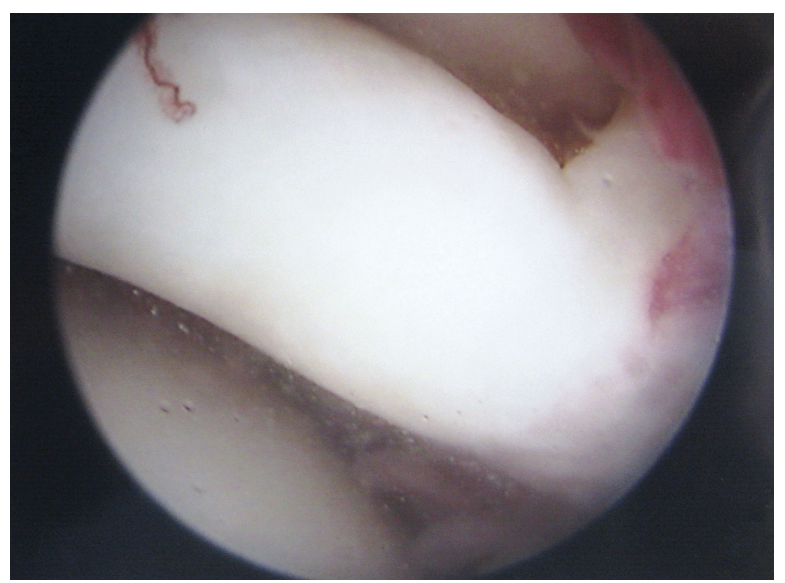

Figure 4. The MM lesion in arthroscopy appeared more extensive 


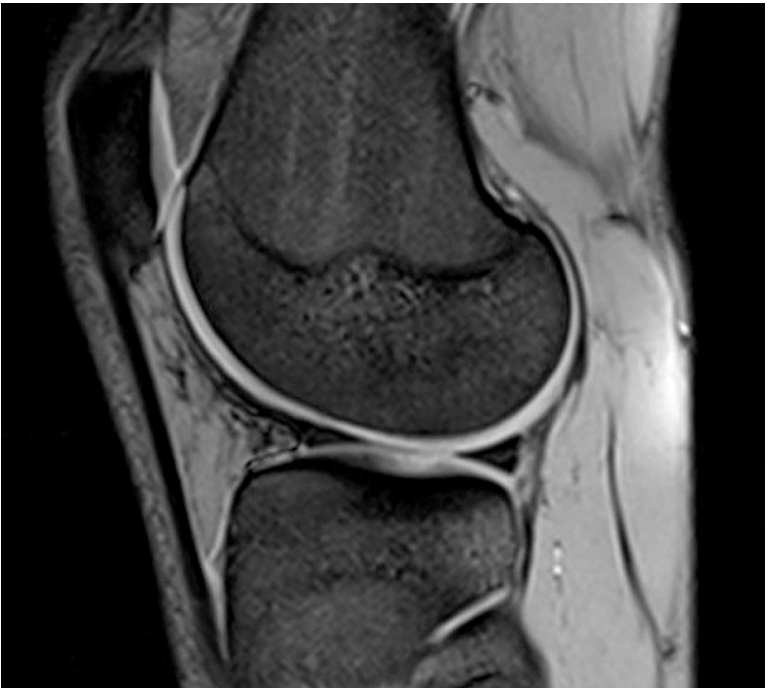

Figure 5. The ML appearance in MRI is normal

The ML lesions are a statistically less frequent problem in knee joint injuries. Sensitivity of both the $\mathrm{OE}$ and MRI is low, but specificity is considerably higher. Kuikka et al. presented similar results [6], sensitivity of $46.7 \%$ and specificity of $98.5 \%$. Research findings in different publications vary markedly [7-9]. Statistical results of MRI for MM and ML clearly differ. Diagnostic sensitivity of MRI for ML is remarkably lower (Figures 5-6). De Smet et al. [11] stated that it is connected with more frequent lesions of the posterior horn of the meniscus or only $1 / 3$ of the meniscus torn back, which is more difficult in diagnostics. The ML tears are a difficult problem in diagnostics and qualification for operative treatment. In the case

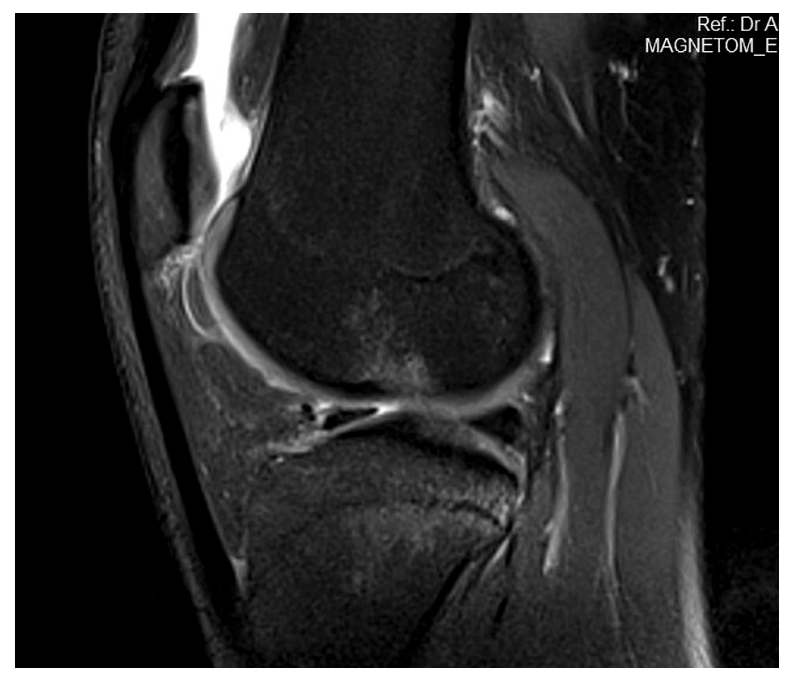

Figure 7. Suspected cartilage lesion

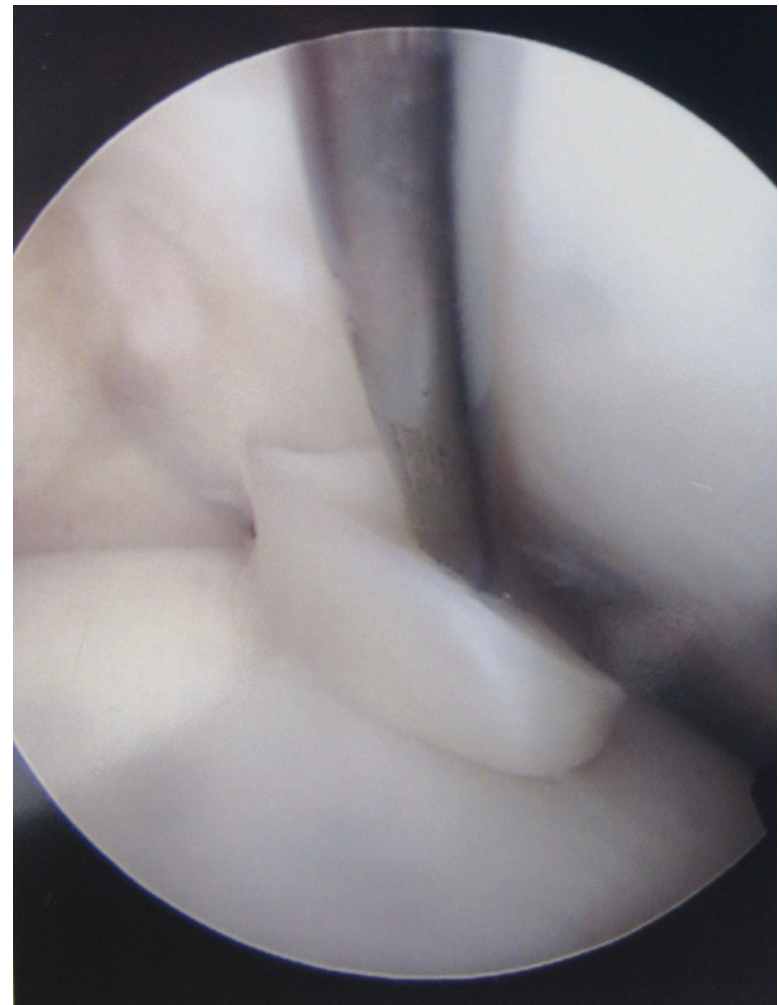

Figure 6. In arthroscopy posterior horn of $\mathrm{ML}$ appeared damaged

of lack of a certain MRI diagnosis, OE is crucial. Injuries of the cartilage are a complicated problem in the diagnostics of knee injuries. In the OE an assessment of the type and lesion location is difficult and MRI does not detect this pathology directly. Analysis of the presented material shows that $\mathrm{OE}$ is more accurate than MRI. Ochi et al. reached similar conclusions [12], underlining MRI's smaller sensitivity. But

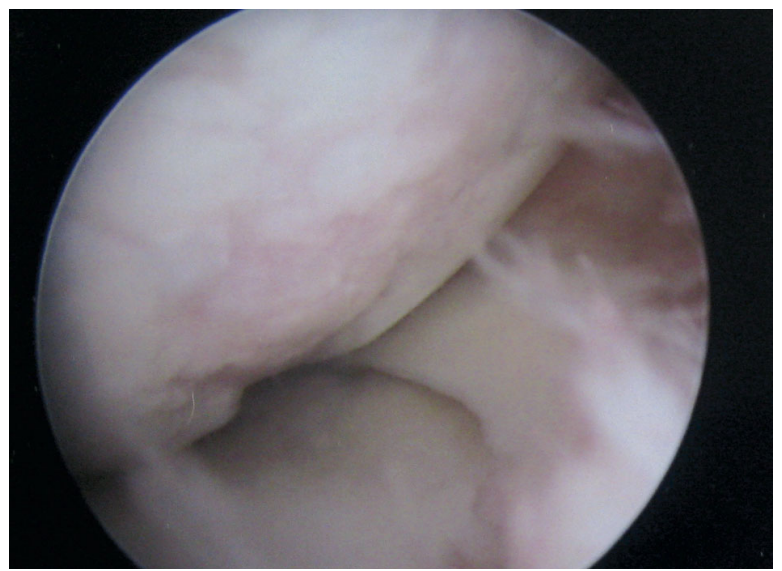

Figure 8. Extensive cartilage defect 
second analysis of MRI after arthroscopy verification changes sensitivity from $40 \%$ up to $71 \%[12,13]$. These observations result from the fact that MRI interpretation concentrates more on detecting meniscal and ligament lesions than on cartilage injuries $[12,13]$. So injuries of the cartilage can be found accidentally in arthroscopy. The OE is more accurate than MRI and it should be a signal for accurate $M R I$ assessment and increasing its preciseness (Figures 7-8).

On account of the low frequency of PCL lesions (1\%), it was not statistically analysed. Based on our own and other authors' experience, both the OE and MRI have great accuracy in detecting this type of lesions $[12,14]$. The diagnostics of knee injuries is a complex problem. We should aspire to increase the accuracy and credibility of the OE and MRI in diagnosing intra-articular lesions. It will reduce the number of diagnostic arthroscopies to the benefit of operating arthroscopies. It will significantly reduce the frequency of diagnostic arthroscopies, lower operating risk and its costs.

\section{References}

1. Lee JK, Yao L, Phelps CT, et al. Anterior cruciate ligament tears: MR imaging compared with arthroscopy and clinical tests. Radiology 1988; 166: 861-4.

2. Buckup K. Clinical tests for the musculoskeletal system: examinations - signs - phenomena Thieme, Germany 2008.

3. Outerbridge RE. The etiology of chondromalacia patellae. J Bone Joint Surg Br 1961; 43-B: 752-7.

4. Rayan F, Bhonsle S, Shukla DD. Clinical, MRI, and arthroscopic correlation in meniscal and anterior cruciate ligament injuries. Int Orthop 2009; 33: 129-32.

5. Terry GC, Tagert BE, Young MJ. Reliability of the clinicalassessment in predicting the cause of internal derangements of the knee. Arthroscopy 1995; 11: 568-76.

6. Kuikka PI, Sillanpää P, Mattila VM, et al. Magnetic resonance imaging in acute traumatic and chronic meniscal tears of the knee: a diagnostic accuracy study in young adults. Am J Sports Med 2009; 37: 1003-8.

7. Fischer SP, Fox JM, Del Pizzo W, et al. Accuracy of diagnoses from magnetic resonance imaging of the knee: a multi-center analysis of one thousand and fourteen patients. J Bone Joint Surg Am 1991; 73: 2-10.

8. Oei EH, Nikken JJ, Verstijnen AC, et al. MR imaging of the menisci and cruciate ligaments: a systematic review. Radiology 2003; 226: 837-48.

9. Ramnath RR, Magee T, Wasudev N, Murrah R. Accuracy of 3-T MRI using fast spin-echo technique to detect meniscal tears of the knee. AJR Am J Roentgenol 2006; 187: 221-5.

10. Rose NE, Gold SM. A comparison of accuracy between clinical examination and magnetic resonance imaging in the diagnosis of meniscal and anterior cruciate ligament tears. Arthroscopy 1996; 12: 398-405.

11. De Smet AA, Mukherjee R. Clinical, MRI, and arthroscopic findings associated with failure to diagnose a lateral meniscal tear on knee MRI. AJR Am J Roentgenol 2008; 190: 22-6.

12. Ochi M, Sumen Y, Kanda T, et al. The diagnostic value and limitation of magnetic resonance imaging on chondral lesions in the knee joint. Arthroscopy 1994; 10: 176-83.

13. Speer KP, Spritzer CE, Goldner JL, Garrett WE Jr. Magnetic resonance imaging of traumatic knee articular cartilage injuries. Am J Sports Med 1991; 19: 396-402.

14. Trieshmann HW Jr, Mosure JC. The impact of magnetic resonance imaging of the knee on surgical decision making. Arthroscopy 1996; 12: 550-5. 